\title{
Quantum Transport in Graphene: Impurity Scattering as a Probe of the Dirac Spectrum
}

\author{
Chuan LI \\ Sophie GuÉron \\ Hélène Bouchiat \\ LPS - Bât. 510 \\ Université Paris Sud \\ CNRS, UMR 8502 \\ 91405 Orsay cedex, France
}

\begin{abstract}
Since the very first investigations of the electronic properties of graphene, the nature of the scattering disorder potential has been shown to play an essential role in determining the carrier density dependence of the conductance. Impurity scattering is characterized by two different times the transport and elastic scattering times which are sensitive to the particular Dirac spectrum of graphene. The analysis of the ratio between these two times gives insight on the nature (neutral or charged) and range of the scatterers. We show how to extract these two times from magneto-transport measurements and analyze their differences in monolayer and bilayer Graphene in relation with the different symmetry properties of their band structure and wave functions. It is found that whereas short range impurity scattering is the dominant mechanism limiting the classical transport, phase coherent mesoscopic transport is very sensitive to long range disorder. In particular, the formation of electron/hole puddles in the vicinity of the charge neutrality point strongly affects the transport of Andreev pairs in the presence of superconducting electrodes. We will also discuss the modification of electronic properties of graphene in the presence of adsorbed atoms and molecules and in particular focus on spin dependent scattering on adsorbates leading to a spin orbit interaction. There is indeed a big interest in controlling and inducing spin orbit interactions in graphene. One can hope to induce and detect a spin Hall effect with a great potential impact in graphene based spintronic devices and ultimately reach a regime of quantum spin Hall physics. In contrast with these very short range scatterers, we discuss the possibility to engineer networks of larger range strained regions in which electronic properties are locally modified by transferring graphene on arrays of silicon oxyde nanopillars.
\end{abstract}

\section{Introduction}

Graphene, the single layer of graphite, is one of the most simple and intriguing 2-dimensional conducting material [1]. The low energy electronic spectrum of graphene is constituted by two Dirac cones centered around the two non equivalent $\mathrm{K}$ points of the Brillouin zone with a perfect electron-hole symmetry at the Fermi level [2]. The electronic wave functions are characterized by their pseudo spin components on the two nonequivalent $\mathrm{A}$ and $\mathrm{B}$ atoms of the hexagonal lattice. The 
symmetry properties of this lattice give rise to a relative phase factor between the 2 spinorial components which depends on the orientation of the Bloch wave vector. This phase factor determines the sensitivity of electronic transport to the disorder potential produced by impurities or charge inhomogeneities. In particular it was emphasized that backward scattering within one valley, is forbidden by these symmetry properties of the wave functions which strongly suppresses Anderson localization in Graphene. The same effect exists also in carbon nanotubes [3]. This is however not true in the presence of very short range impurities such as single atom vacancies which induce intervalley scattering.

One way to tackle the scattering mechanism in graphene is to compare the two different scattering times: (i) the transport time $\tau_{t r}$, which governs the current relaxation and enters the Drude conductivity $(\sigma)$, (ii) the elastic scattering time $\tau_{e}$, which is the lifetime of a plane wave state [4]. Since $\tau_{t r}$ and $\tau_{e}$ involve different angular integrals of the differential cross section, they differ as soon as the Fourier components of the potential depend on $q$. A large ratio $\tau_{t r} / \tau_{e}$ indicates that scattering is predominantly in the forward direction, so that transport is not affected much by this type of scattering. This is the case in 2D electron gases (2DEG) confined in GaAs/GaAlAs heterojunctions with the scattering potential produced by remote charged Si donors [5], where $\tau_{t r} / \tau_{e}$ is larger than 10. We show how to extract these two times from magnetotransport. These experiments are discussed in the first part of the paper. The ratio $\tau_{t r} / \tau_{e}$ is found to be close to 1.8 and nearly independent of the carrier density. Comparison with theoretical predictions suggests that the main scattering mechanism which dominates the physics of classical transport between 1 and $10 \mathrm{~K}$ is due to strong (resonant) scatterers of a range shorter than the Fermi wavelength.

The second part of the paper is devoted to quantum mesoscopic transport at very low temperature in phase coherent samples. It is dominated by interference effects between all wave packets crossing the sample. This interference pattern is sensitive to variations in disorder configuration, Fermi energy or magnetic flux, leading to reproducible conductance fluctuations as one of these parameters is changed.

These fluctuations which are the trade mark of mesoscopic transport have been investigated in mesoscopic graphene samples and characterized them by their correlations and amplitudes as a function of Fermi energy and magnetic field. This analysis has revealed a strong sensitivity of conductance fluctuations to the long range disorder induced by the formation of charge inhomogeneities (electron hole puddles) [6]. Another way to probe the coherent nature of quantum transport in mesoscopic systems is to investigate proximity induced superconductivity in the presence of superconducting electrodes. In particular the induced Josephson supercurrent has been shown to decay on the thermal and phase coherence lengths scales $L_{T}$ and $L_{\varphi}$ characteristic of mesoscopic transport. Supercurrents could be successfully measured in short graphene Josephson corresponding to $L<\xi_{S}$ where $\xi_{S}$ is the superconducting coherence length $[7,8,9,10]$. We present also measurements in the long junction limit $L>>\xi_{S}$. Whereas a supercurrent is clearly observed far from the neutrality point, its value is strongly suppressed close to the neutrality point. These results point the role of low carrier density puddles in the suppression of the supercurrent along which Andreev pairs are specularly reflected.

The third part of this paper is essentially prospective. Beyond the investigation of impurity scattering in graphene, we discuss the possibility of designing new func- 
tionalities in graphene by controlling the nature of intentionally deposited scattering centers. We will first consider the case of add-atoms, small aggregates or molecules chosen for their high spin orbit scattering properties. The main motivation is to induce spin orbit interactions in graphene. One can hope to induce and detect a gate dependent spin Hall effect with a great potential impact in graphene based spintronic devices and ultimately reach a regime of quantum spin Hall physics. It has recently been shown that the measurements of transport and elastic times discussed in section II provide a quantitative determination of the spin orbit strength of added scatterers. Following the experiments of Crommie et al. [11] which demonstrated that local strain induces a polarization of the pseudo spin degrees of freedom acting as an effective magnetic field, we will show that it is also possible to create local strained regions by deposition of graphene on etched substrates consisting of arrays of sharp silicon oxide nanopillars. We expect electronic properties to be strongly modified in the strained graphene regions with an increase of the density of states at the Dirac point.

\section{Impurity scattering in graphene: determination of the transport and elastic scattering times}

The nature of impurity scattering in graphene has been the subject of intense debate these last years. In particular the quasi-linear carrier density dependence of the conductivity $\sigma\left(n_{c}\right)$ cannot be understood when simply considering neutral short range impurities with "white noise" (wavevector $q$ independent) scattering: indeed the associated inverse scattering time varies linearly with the density of states leading to a conductivity independent of the carrier density. Including intervalley scattering $[3,12,13]$ leads only to a weak (logarithmic) dependence of $\sigma\left(n_{c}\right)$.In contrast, scattering on charged impurities originates from a $q$ dependent screened Coulomb potential described in the Thomas-Fermi approximation [14, 15, 16]. This leads to a linear $\sigma\left(n_{c}\right)$. However experiments performed to probe this question measured the change in $\sigma$ upon immersion of graphene samples in high dielectric constant media find a dependence of the conductance with the dielectric constant which is much too weak to be understood by charged impurity scattering [17], see also the more recent work on $\mathrm{SrTiO}_{3}$ substrates [18]. Alternate explanations involve resonant scattering centers with a large energy mismatch with the Fermi energy of carriers [13, 19]. We will see that these strong scattering centers play indeed the major role in the determination of the classical conductivity. Impurity scattering is characterised by the transport and elastic scattering times which are related to the following angular integrals of the scattering crossection $\sigma(\theta)$ :

$$
\begin{aligned}
& 1 / \tau_{e}=n_{i m p} v_{F} \int d \theta \sigma(\theta), \\
& 1 / \tau_{t r}=n_{i m p} v_{F} \int d \theta(1-\cos \theta) \sigma(\theta) .
\end{aligned}
$$

For isotropic relatively short range scatterers of range $R$ smaller than the Fermi wave length but longer than the interatomic distance $a$ which forbids intervalley scattering, the angular dependence dependence of the scattering cross section $\sigma(\theta)$ contains an angular dependence in $\sigma_{0} \frac{1+\cos j \theta}{2}$ the Berry phase factor $j \theta$ is specific to the symmetry of the wave functions within a single valley. One has $j=1$ for the graphene monolayer leading to the absence of backscattering which is also at the origin of Klein tunneling. On the other hand $j=2$ for the bilayer. As a result from 
Eq. (1) one expects the ratio $\tau_{t r} / \tau_{e}$ to be equal to 2 for the monolayer and 1 for the bilayer.

In the following we discuss how these transport and elastic scattering times can be determined from magnetoresistance experiments. In high magnetic field, when the cyclotron frequency is larger than $1 / \tau_{e}$, the magneto-conductivity exhibits Shubnikov de Haas (ShdH) oscillations related to the formation of Landau levels. The broadening of these levels at low temperature yields $\tau_{e}$, while the low field quadratic magneto-conductivity yields $\tau_{t r}$. The samples investigated were fabricated by exfoliation of natural graphite flakes and deposition on a doped silicon substrate with a $285 \mathrm{~nm}$ thick oxide. The carrier density can be tuned from electrons to holes through the charge neutrality point by applying a voltage on the backgate. The ML and BL samples were identified using Raman spectroscopy. The electrodes were fabricated by electron beam lithography and either sputter deposition of $40 \mathrm{~nm}$ thick palladium or Joule evaporation of a bilayer $5 \mathrm{nmTi} / 70 \mathrm{~nm}$ Au. The low temperature magnetoresistance (MR) is shown on Fig. 1 for 2 different samples, a monolayer and a bilayer. It exhibits a quadratic behavior at low field whereas Shubnikov de Haas oscillations appear at high field. We now describe how we extract $\tau_{t r}$ and $\tau_{e}$ from this data (see Fig. 1). The two-terminal MR results from mixing of the diagonal $\left(\rho_{x x}\right)$ and off-diagonal $\left(\rho_{x y}\right)$ components of the resistivity tensor $[21,22]$. The degree of mixing depends on the aspect ratio of the sample. For a square geometry, close to that of the monolayer, $R(B)=\left[\rho_{x x}^{2}+\rho_{x y}^{2}\right]^{1 / 2}$; in a short wide sample such as the bilayer $R(B)=(L / W)\left[\rho_{x x}^{2}+\rho_{x y}^{2}\right] / \rho_{x x}$. Intermediate geometries can be calculated following the model developed in [21]. It is then possible to reconstruct the complete MR from the expressions of the resistivity tensor [23] valid in the limit of moderate magnetic field where ShdH oscillations can be approximated by their first harmonics:

$$
\begin{aligned}
& \delta \rho_{x x}(B) / \rho_{0}=4 D_{T} \exp \left[-\frac{\pi}{\omega_{c} \tau_{e}}\right] \cos \left[\frac{j \pi E_{F}}{\hbar \omega_{c}}-\phi\right] \\
& \rho_{x y}(B)=\rho_{0} \omega_{c} \tau_{t r}-\delta \rho_{x x}(B) / 2 \omega_{c} \tau_{t r},
\end{aligned}
$$

where $\rho_{0}=1 / \sigma$ is the zero-field resistivity and $\omega_{c}=e B / m^{*}$ is the cyclotron frequency, $m^{*}=\hbar k_{F} / v_{F}$ is the cyclotron mass which depends explicitly on the Fermi wave vector $k_{F}$ for the ML (constant Fermi velocity). On the other hand, the bilayer's dispersion relation is parabolic at low energy and $m^{*}$ can be approximated by the effective mass $m_{e f f}=0.035 m_{e}$, nearly independent of the carrier density in the range of $V_{g}$ explored where $\left|E_{F}\right| \leq 80 \mathrm{meV}$ is smaller by a factor 5 than the energy band splitting [1]. The phase $\phi$, either $\pi$ or $2 \pi$, and the parameter $j$, either 1 or 2 , depend on the nature of the sample (ML or BL) The Fermi energy $E_{F}$ is $\hbar k_{F} v_{F}$ for the monolayer and $\hbar^{2} k_{F}^{2} /\left(2 m_{e f f}\right)$ for the bilayer. The prefactor $D_{T}=\gamma / \sinh (\gamma)$ with $\gamma=2 \pi^{2} k_{B} T / \hbar \omega_{c}$ describes the thermal damping of the oscillations.

To analyze the data we first deduce $k_{F}$ from the periodicity of the ShdH oscillations function of $1 / B$. This determination is more reliable than the estimation of $n_{c}=k_{F}^{2} / \pi$ from the gate voltage and the capacitance between the doped silicon substrate and the graphene sample. This is specially the case close to the neutrality point where the sample is possibly inhomogeneous [20]. Knowing $k_{F}$ we then determine $\tau_{t r}$ from the low field quadratic magnetoresistance which is found to be independent of temperature between 1 and $4 \mathrm{~K}$ :

$$
R(B)-R(0)=\frac{h}{2 e^{2}} \frac{L}{W} \frac{1}{k_{F} v_{F} \tau_{t r}} \alpha_{g}\left(\omega_{c} \tau_{t r}\right)^{2} .
$$



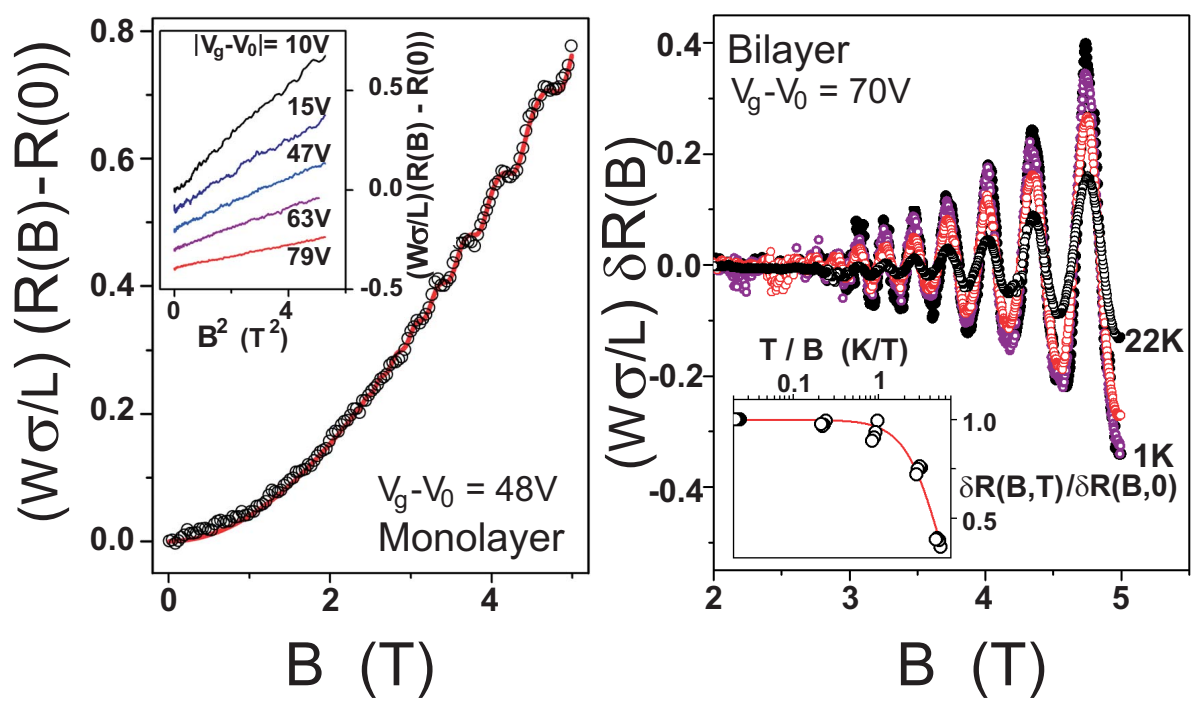

Figure 1: Analysis of the magnetoresistance. Left panel: Magnetoresistance of a monolayer (sample A). Dots: experimental points at $T=1 \mathrm{~K}$; Continuous line: fit according to Eqs. (3) and (2). Inset: $B^{2}$ dependence of the low-field magnetoresistance for different gate voltages (Curves shifted along the $\mathrm{Y}$ axis for clarity). $\tau_{t r}$ is extracted from the slopes of these curves according to Eq. (3). Notice that the slope increases in the vicinity of the Dirac point reflecting the divergence of the inverse effective mass. Right panel: ShdH oscillations of the longitudinal component of the resistivity in a bilayer sample B for different temperatures after subtraction of the quadratic background. The Fermi wavevector $k_{F}$ and the elastic time $\tau_{e}$ are deduced from the period and the decay of the oscillations with $1 / B$ at low temperature. Inset: Temperature dependence of the oscillations amplitude normalized to $T=0$. Solid line: fit according to the Lifshitz-Kosevich formula $D_{T}=$ $\gamma / \sinh (\gamma)$ with $\gamma=2 \pi^{2} k_{B} T / \hbar \omega_{c}$ [23]. The effective mass determined from this fit is $m_{e f f}=$ $0.035 \pm 0.002 m_{e}$ in the whole range of gate voltage investigated.

We have used the relation $\sigma=\rho_{0}^{-1}=\left(2 e^{2} / h\right) k_{F} v_{F} \tau_{t r}$. The dimensionless coefficient $\alpha_{g}$, which depends on the aspect ratio of the sample was determined numerically following [21] and the experimental values of $W$ and $L$. It is important to note that this determination of $\tau_{t r}$ is independent of any assumption of the contact resistance on two terminal samples. We finally extract $\tau_{e}$ from the damping of the first harmonic of ShdH oscillations in the resistivity tensor in $\exp (-\beta / B)$ where $\beta=\pi \hbar k_{F} / e v_{F} \tau_{e}$, see Eq. (2).

The $k_{F}$ dependences of $\tau_{t r}$ and $\tau_{t r} / \tau_{e}$ are shown in Fig. 3 for several two terminal samples A, B and C and two multi-terminal samples (D and E) with Hall-bar geometry (see [24] for more details). We observe different behaviors for the ML samples, where $\tau_{t r}$ has a minimum at the CNP, and the BL, where it has a maximum. In all cases, despite rather large variations of $\tau_{t r}, \tau_{t r} / \tau_{e}$ is nearly independent of $k_{F}$. It is equal to $1.7 \pm 0.3$ for the monolayers $\mathrm{A}, \mathrm{C}, \mathrm{E}$ and to $1.8 \pm 0.2$ for the bilayer in the whole range explored, which corresponds to $n_{c}$ between $1.5 \times 10^{11}$ and $5 \times 10^{12} \mathrm{~cm}^{-2}$. As already discussed, that $\tau_{t r} / \tau_{e}$ is of the order but smaller than 2 indicates that the typical size of the scatterers does not exceed the Fermi wavelength for the ML samples. According to Eq. (1), the factor 2 instead of 1 expected for short range impurities in GaAs is due to the symmetry of the wave functions within one valley more precisely to the $\pi$ Berry phase factor which forbids backscattering within one 


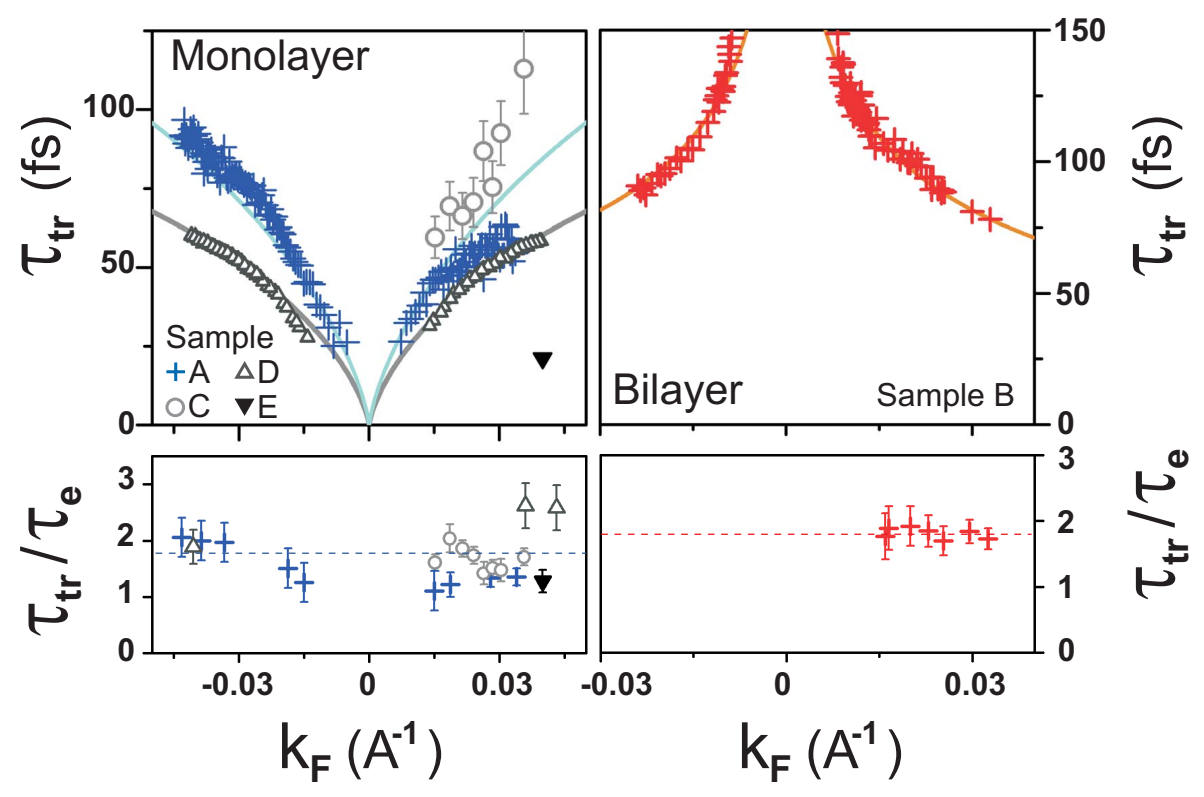

Figure 2: $k_{F}$ dependence of $\tau_{t r}$ and $\tau_{t r} / \tau_{e}$ ratio. Left panel: monolayers A, C, D and E. Right panel: bilayer B. The continuous lines are the fits for samples A, B and D according to the resonant impurity model, Eq. (4). For samples A B and C (two terminal configuration) $\tau_{t r}$ was extracted from the low field magnetoresistance (crosses) whereas it was extracted from the zero field conductivity for samples D and E. Positive/negative values of $k_{F}$ correspond to electron/hole doping. Lower panels: ratio $\tau_{t r} / \tau_{e}$ where $\tau_{e}$ is deduced from the fit of the low temperature decay of the ShdH oscillations. Dotted lines figure the average value $\tau_{t r} / \tau_{e}=1.8$. We note that sample $\mathrm{D}$ exhibits a value of $\tau_{t r} / \tau_{e}$ at high electron doping which is larger than $2(\simeq 2.4)$. The area of this sample $\left(12 \mu \mathrm{m}^{2}\right)$ is much larger than the area $\left(\simeq 1 \mu \mathrm{m}^{2}\right)$ of all the other samples A, B, C and E. We suspect that this large sample contains more spatial inhomogeneities than the other smaller samples which could explain a reduced value of $\tau_{e}$.

valley. A smaller ratio would have been however expected for the BL, since symmetry of wave functions do not forbid intravalley backscattering in that case. We will see in the following that this ratio $\tau_{t r} / \tau_{e}$ can be used to probe the presence of spin orbit scattering impurities which modify the symmetry of the wave functions in graphene.

We now compare our results on $\tau_{e}$ and $\tau_{t r}$ to theoretical predictions. We first consider scattering on charged impurities [14, 15]. Screened charged impurities are characterized by a screening radius $1 / q_{s c}$, which in the Thomas-Fermi approximation, is given by $1 / q_{T F} \equiv \pi \epsilon \hbar v_{F} / e^{2} k_{F}$, where $\epsilon$ is the appropriate dielectric constant. In the Born approximation, the transport time is $\tau_{t r} \propto q_{s c}^{2} v_{F} / k_{F}$. For a monolayer, $q_{T F} / k_{F}$ is a constant $\simeq 3$ and both $\tau_{t r}$ and $\tau_{e}$ are then expected to increase as $k_{F}$, which is not what we observe in Fig. 3 where the increase is sublinear. The disagreement is even stronger for a bilayer, where the ratio $q_{T F} / k_{F} \propto 1 / k_{F}$ varies between 3 at high doping and 12 close to the neutrality point. The transport time is then expected to vary linearly with $n_{c}$, if the screening radius is estimated as $\sim 1 / k_{F}$, or to be independent of $k_{F}$ if estimated as $\sim 1 / q_{T F} \ll 1 / k_{F}$ [15], neither of which agrees with our data, see Fig. 3.

An alternative explanation is resonant scattering resulting from vacancies or any 
other kind of impurities of range $R$ such that $a \lesssim R \ll 1 / k_{F}$, where $a$ is the carboncarbon distance, and with a large potential energy $[13,19,24]$. It is characterized by a transport cross section

$$
A_{t r} \simeq \frac{\pi^{2}}{k_{F} \ln ^{2}\left(k_{F} R\right)} .
$$

The resulting transport time $\tau_{t r}=1 /\left(n_{i} v_{F} A_{t r}\right)\left(n_{i}\right.$ is the concentration of impurities) leads to a conductance increasing as $n_{c}$ with logarithmic corrections for both the ML and BL. In both cases, our extracted $\tau_{t r}\left(k_{F}\right)$ (see Fig. 3) are compatible with the square logarithmic dependence of Eq. (4).

This analysis also corroborates our results on the ratio $\tau_{t r} / \tau_{e}$ indicating scatterers with a range smaller than the Fermi wavelength (but possibly of the order of or slightly larger than the lattice spacing). Whereas the resonant character is not essential for the validity of Eq. (4) for massive carriers (corresponding to the bilayer) [26], it has been shown that it is essential for massless carriers in the monolayer [27]. This resonant-like character, although not straightforward has been demonstrated in the case of scattering centers created by vacancies in graphene over a wide range of Fermi energies [28]. As shown in detail in [24], it is not necessary to fine-tune $k_{F}$ to obtain the $\ln ^{2}$ dependence in Eq. (4).

In conclusion to this section, our results indicate that the main scattering mechanism which determines the classical transport properties in our graphene samples is due to strong neutral defects, with a range shorter than the Fermi wavelength and possibly of the order of $a$, inducing resonant (but not unitary) scattering. Likely candidates are vacancies, as observed in transmission electron microscopy [29], voids of several atomic size, ad-atoms or short range ripples as suggested in [30] [31]. It was pointed out that these ripples also give rise to intravalley dephasing with a characteristic time which can be deduced from weak localisation measurements [32].

\section{Quantum transport: proximity induced superconductivity and specular An- dreev reflection.}

We now move to the low temperature transport measured below $1 \mathrm{~K}$ in the phase coherent regime where the length of the samples investigated does not exceed the characteristic length for mesoscopic transport $L_{\varphi}$ and $L_{T}$, the phase coherence and thermal length. We have chosen to focus on the situation where graphene is connected to superconducting electrodes giving rise to the formation of Andreev pairs which are coherent superposition of time reversed electron hole pairs. It was shown that the formation of these Andreev pairs could be strongly affected by the nature of the symmetric electron-hole band structure of graphene and be very sensitive to charge inhomogeneities in vicinity of the neutrality point [34]. Indeed, transport across a Superconductor/Normal metal $(\mathrm{S} / \mathrm{N})$ interface at subgap energy implies extracting two electrons from the superconductor and injecting them into the $\mathrm{N}$, which produces a correlated Andreev pair in the normal metal. In a usual normal metal, which is highly doped in the sense that the Fermi level lies well within the conduction band, both electrons are injected in the conduction band of the $\mathrm{N}$. The two injected members of the Andreev pair then follow the same, albeit timereversed, diffusive path in the normal conductor, so that coherent propagation can occur over several micrometers (the phase coherence length at low temperature). 

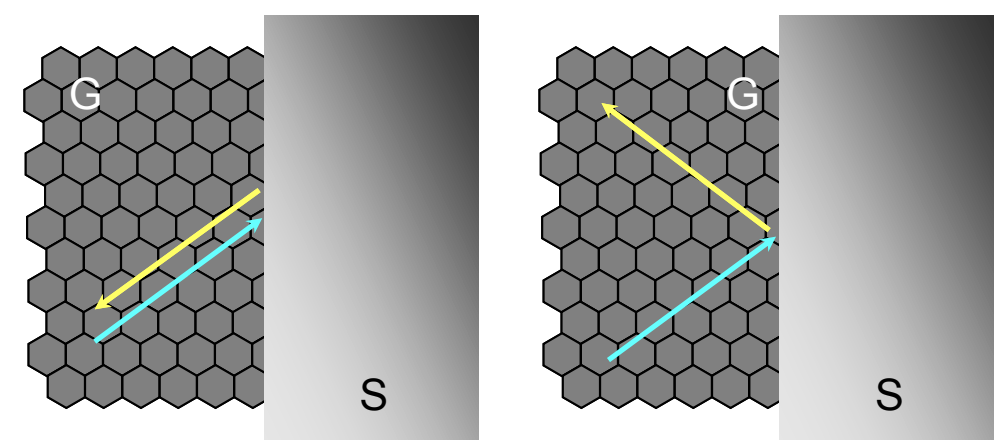

Figure 3: Sketch of the retro- and specular Andreev reflection at a G/S interface. Left: Retroreflection occurs in usual conductors and in doped graphene, where the Fermi energy much exceeds the superconducting electrode's energy gap $\Delta, E_{F} \gg \Delta$. Right: The specular Andreev reflection occurs in graphene at doping small enough that $E_{F} \ll \Delta$.

This coherent propagation leads to supercurrents that flow through such normal conductors several microns long connected to two superconductors. In contrast, at a superconductor/graphene $(\mathrm{S} / \mathrm{G})$ interface, if the superconductor's Fermi level is aligned with the graphene Dirac point, the two electrons of a Cooper pair must split into an electron in the conduction band and the other in the valence band. The two members of the injected pair in the graphene now have the same velocity (rather than opposite) parallel to the S/G interface (see Fig. 3) and thus do not follow the same diffusive path. The observation of this special type of pair injection, also called "specular Andreev reflection", has so far remained elusive. This is because the doping inhomogeneities in the graphene samples, of several milli-electronvolts [33], are much larger than the superconductor's energy gap. Thus only the usual injection of counter-propagating electron pairs (also called Andreev retroreflection) sets in.

In the following we show that diffusive transport of Andreev pairs through quantum coherent graphene reveals an analog of specular Andreev reflection at an $\mathrm{S} / \mathrm{G}$ interface, in the form of specular reflections of Andreev pairs at the interface between a doped charge puddle and a zero density region. These processes result in the destruction of counter-propagation upon specular reflection, and lead to a large phase accumulation within each Andreev pair. Since all pairs contribute to the supercurrent with their phase, the resulting supercurrent is suppressed. We argue that this specular reflection explains the suppression of the critical current that we observe near the Charge Neutrality Point (CNP) in quantum coherent, long and diffusive SGS junctions see Fig. 4 . Gate tunable Josephson junctions have been investigated by a large number of groups, we focus in the following on the behavior of long $\left(L \gg \xi_{S}\right)$ compared to short $\left(L \ll \xi_{S}\right)$ junctions where $\xi_{S}$ is the superconducting coherence length. We compare data obtained on a long junction $1 \mu_{m}$ long graphene samples connected to $\mathrm{Pd} / \mathrm{Nb}$ electrodes and a short junction $0.3 \mu \mathrm{m}$ long with $\mathrm{Ti} / \mathrm{Al}$ electrodes. In the theory of the proximity effect the critical current of a short junctions is $I_{c}=\Delta / e R_{N}$ whereas in the diffusive, long junction limit, the critical current has a maximum zero temperature value given by the Thouless energy $E_{T h}$ divided by the normal resistance state $R_{N}$, multiplied by a numerical factor $\alpha$ which depends on the junction length $L: I_{c}=\alpha E_{T h} / e R_{N}$, where 


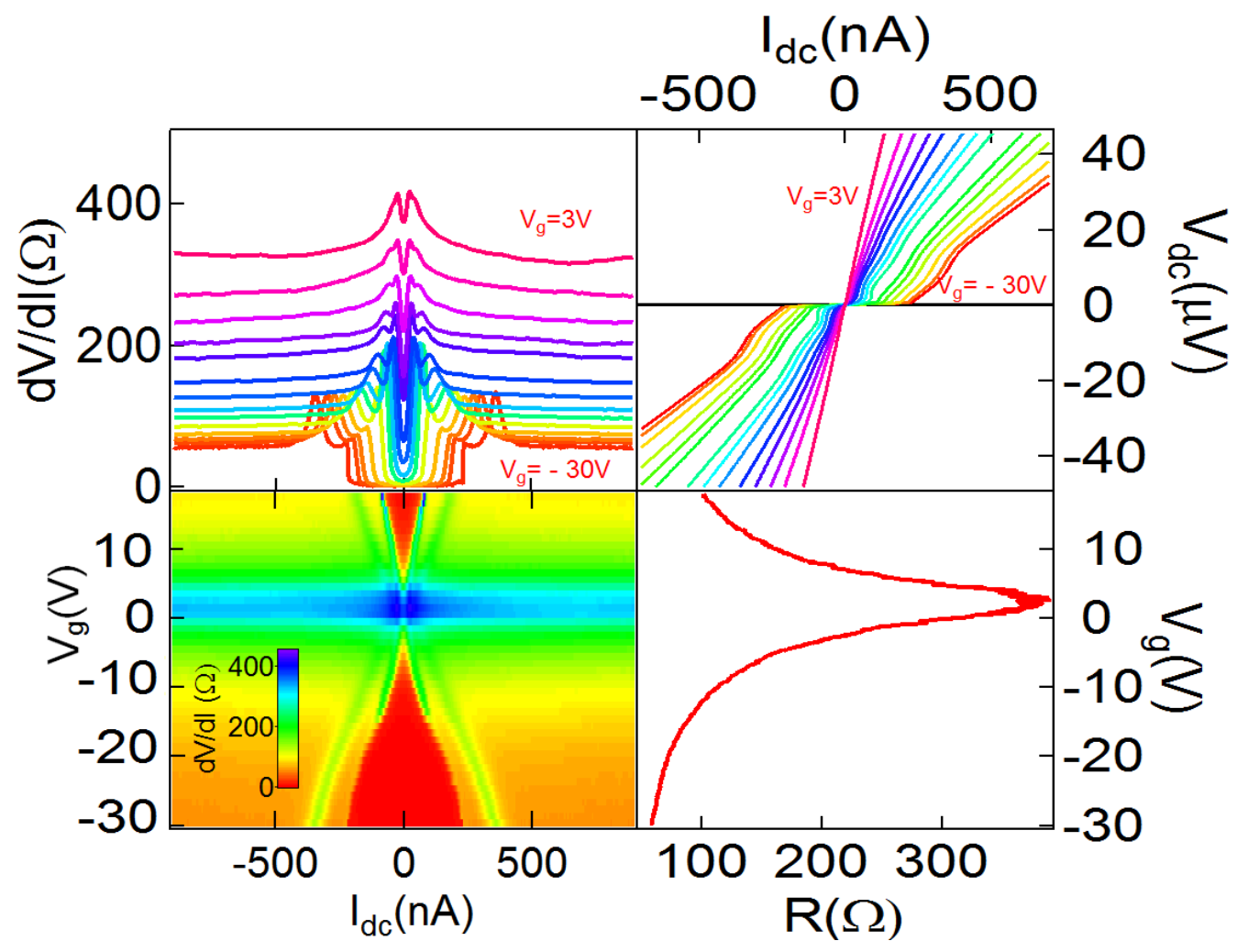

Figure 4: Proximity effect in graphene connected to Nb electrodes at $200 \mathrm{mK}$. Upper left panel: $d V / d I$ vs $I_{\mathrm{dc}}$ for different gate voltages, and, bottom left panel, its 2 dimensional color plot. The suppression of critical current in a gate voltage region of $\pm 10 \mathrm{~V}$ around the charge neutrality point is noticeable. Upper right panel: $\mathrm{I}(\mathrm{V})$ curves for different gate voltages, showing how the proximity effect varies between a full proximity effect with zero resistance at high doping, and quasi normal behavior with a linear IV around the charge neutrality point. Lower right panel: Zero bias differential resistance as a function of gate voltage in the normal state, from which the $R_{N}$ is determined. A small magnetic field was applied to destroy the constructive interference leading to the supercurrent. From Komatsu et al. [36].

$E_{T h}=\hbar D / L^{2}$, with $D$ is the diffusion constant.

The tunability of graphene is an asset to probe these relations. As shown in Fig. 5, one can compare the measured switching current for the short and long junctions investigated to the superconducting gap (respectively the Thouless energy) divided by the normal state resistance as the gate voltage is varied. It is clear from Fig. 5 that there is not a constant factor between $E_{T h} / e R_{N}$ and $I_{c}$ for the long junction but that $I_{c}$ is strongly suppressed at small gate voltage, as the charge neutrality point is approached. Note that this suppression is not observed in the short SGS junction. It was neither been reported in the other graphene based SNS junctions, measured in other groups which are more than two or three times shorter than the long devices we have investigated.

We attribute this suppression close to the CNP to specular reflection of an Andreev pair at the charge puddle contours, as sketched in Fig. 6. Indeed, around 

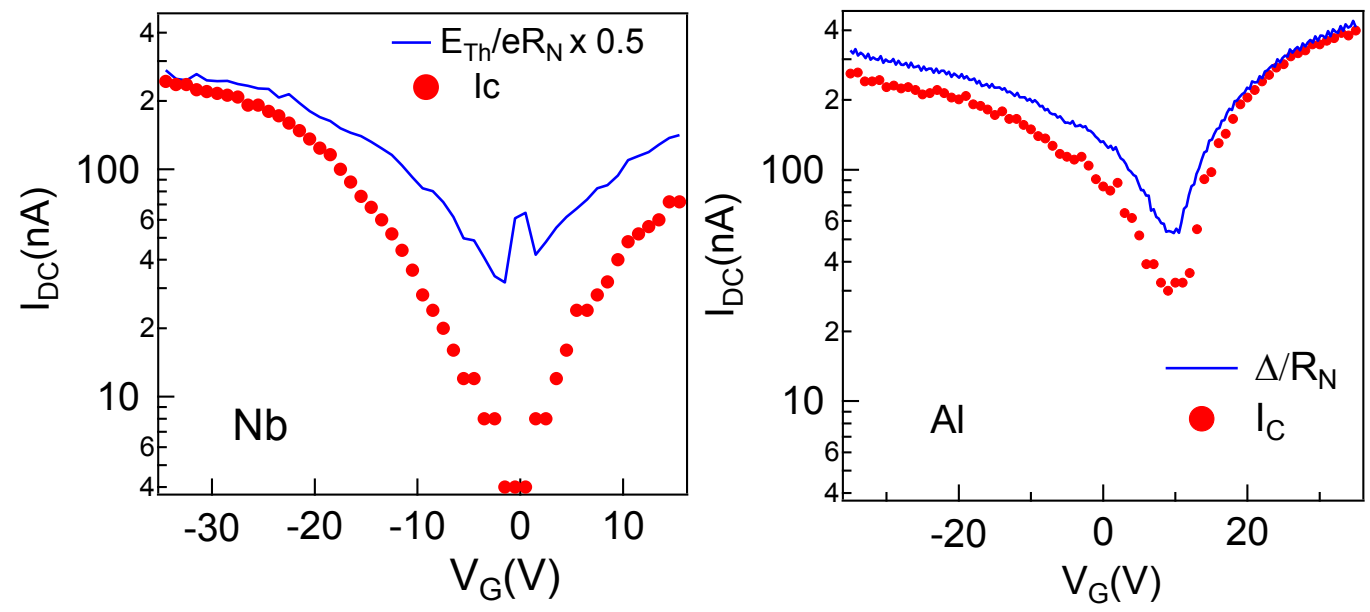

Figure 5: Comparison between the gate voltage dependence of the critical current of the long $\mathrm{Nb} / \mathrm{G} / \mathrm{Nb}$ junction with the gate voltage dependence of the critical current of the short $\mathrm{Al} / \mathrm{G} / \mathrm{Al}$ junction compared to the theoretical predictions. For the long junction we used two ways of defining the switching current: $I_{\mathrm{c}}$, the largest current for which the differential resistance $d V / d I$ is zero, and $I_{\mathrm{c}}^{*}$, the inflection point of the jump in $d V / d I$ towards large resistance. As the Dirac region is approached, the switching current $I_{c}$ reaches zero but a steep resistance increase still occurs at $I_{c} *$. The critical current of the short junction follows the expected value $\delta / R_{N}$.

\section{Doped diffusive graphene}
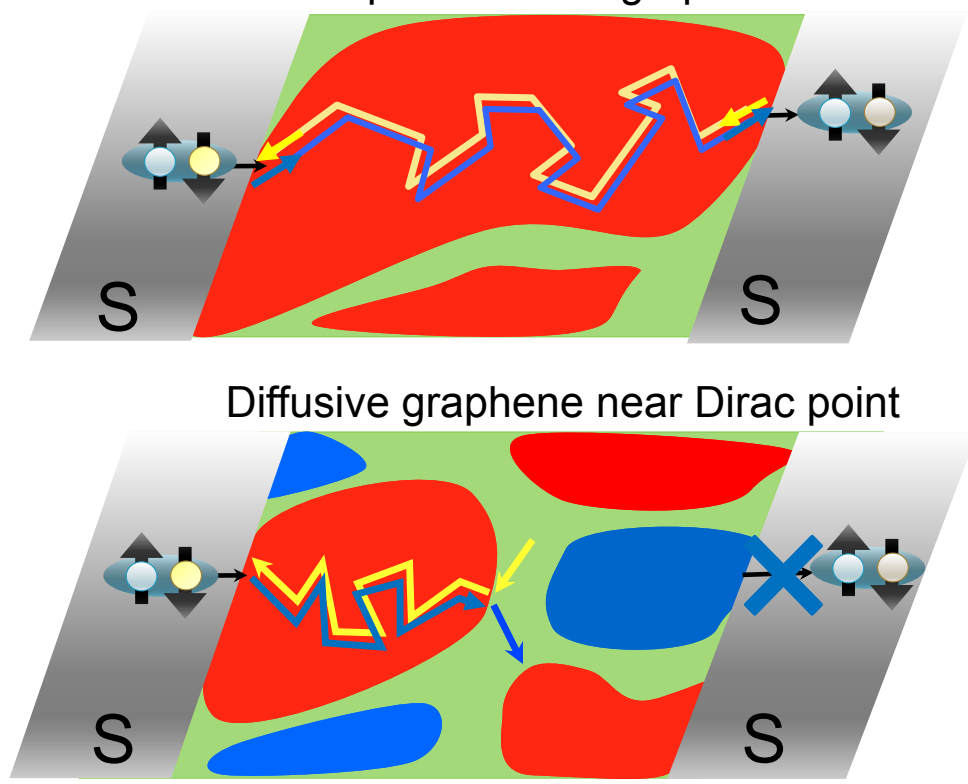

Figure 6: Sketch of the superconducting proximity effect through diffusive graphene, at high and low doping. Top sketch: Highly doped regime. The usual Andreev retroreflection at the S/G interface leads to diffusive counterpropagation with zero total phase accumulation. Bottom sketch: Low doping regime. Specular Andreev reflection of propagating Andreev pairs can occur at an n/0 or $\mathrm{p} / 0$ junction, leading to loss of counterpropagation and thus large phase accumulation within an Andreev pair. Supercurrent, which results from all Andreev trajectories, is destroyed. The red region is electron doped, the blue one hole doped, and the green region in between has zero doping $\left(E_{F}<E_{T h}\right)$. 
the CNP, electron-doped regions coexist with hole doped ones, forming a network of so called puddles [33]. Where the doping varies from $\mathrm{n}$ to $\mathrm{p}$ doping there is necessarily a boundary with exactly zero doping, to within $k_{B} T$, termed a 0 region. Thus a time-reversed Andreev pair formed by the usual Andreev retroreflection at the superconductor/graphene interface has, near the CNP, a large probability of encountering a $\mathrm{n} / 0$ or $\mathrm{p} / 0$ boundary. At such boundaries such junctions, a specularlike reflection must occur when two counter propagating electrons diffusing in the n-doped region are converted into two electrons belonging to two different bands in the 0 region. The change in relative velocity destroys the counter propagation of the pair. As the two electrons diffuse across the rest of the graphene, they undergo uncorrelated scattering events and their relative phase difference increases. Since the total supercurrent is the sum of all contributions from the propagating Andreev pairs, constructive interference is destroyed when counter-propagation is lost, and thus the supercurrent is suppressed (see fig. 6). Interestingly, the effect of these puddles is immense in the superconducting state (and presumably all the more so that the superconducting coherence length, the "size" of the pair, is small with respect to the puddle size), whereas it is much weaker in the normal state where thanks to Klein tunneling, the puddles do not suppress single quasiparticle propagation so much.

In summary, whereas the specular Andreev reflection in ballistic $\mathrm{S} / \mathrm{G} / \mathrm{S}$ junctions can yield a supercurrent [35], we have shown that in diffusive $\mathrm{S} / \mathrm{G} / \mathrm{S}$ junctions a specular-like reflection of Andreev pairs at $\mathrm{p} / 0$ or $\mathrm{n} / 0$ junctions leads to accumulation of phase difference within the Andreev pair. The critical current is then suppressed, in a manner which depends on the number of such $\mathrm{n} / 0$ (or $\mathrm{p} / 0$ ) junctions within the sample. This translates into a critical current suppressed most near the charge neutrality point. The supercurrent suppression by charge puddles is thus expected to be largest in samples that are long (large ratio of sample length $L$ to puddle size, typically larger than $50 \mathrm{~nm}$ [33]) and connected to superconductors with large gaps, corresponding to smaller superconducting coherence lengths $\left(\xi_{s}=\sqrt{\hbar D / \Delta}\right.$ is typically $125 \mathrm{~nm}$ in graphene for $\mathrm{Nb}(\Delta=1.6 \mathrm{meV})$ or $170 \mathrm{~nm}$ for $\operatorname{ReW}(\Delta=1.2 \mathrm{meV}$, as compared to $350 \mathrm{~nm}$ for $\mathrm{Al}(\Delta=0.2 \mathrm{meV})$, given the diffusion constant $D=4.10^{-2} \mathrm{~m}^{2} / \mathrm{s}$ in these graphene samples.

\section{Perspectives: inducing new functionalities in graphene by creating (on pur- pose) scattering centers}

We have discussed in the previous sections how impurity scattering and charge inhomogeneities affects transport properties of disordered graphene. We will now discuss to what extent it is possible to induce new electronic properties in graphene by functionalisation with scattering centers which can be of very different nature.

\subsection{Adatoms, agregates or molecules}

It has been already shown that charged impurities give rise to anomalies in the density of states depending on the position of the Fermi energy. When the charge is larger than a critical value these evolve into huge peaks identified as "collapse" states by analogy with the capture of relativistic electrons by large Z positively charged nucleus [37]. It has also been predicted that magnetic adatoms can give rise 
to gate dependent Kondo screening and exchange coupling with electron or holes carriers in graphene $[38,39]$. This gate induced magnetism has been observed with the deposition of molecules on graphene [40]. Another very interesting functionality which can be induced in graphene is spin orbit coupling. It was shown indeed that the band structure of graphene with spin orbit interactions can be completely modified with the opening of a gap at the Dirac points ad the formation of topological spin polarized edge states. The intrinsic spin orbit of carbon atoms being extremely small, it has been proposed to deposit ad atoms on graphene. Depending on the strength of their chemical bounding and their positions with respect to the hexagonal lattice these atoms can either induce a so called "intrinsic" spin orbit or a Rashba interaction described respectively by the hamiltonians $H_{S O}$ and $H_{R}$ :

$$
\begin{aligned}
& H_{S O}=\Delta_{S O} \tau_{z} \sigma_{z} s_{z} \\
& H_{R}=\Delta_{R}\left(\tau_{z} \sigma_{x} s_{y}-s_{x} \sigma_{y}\right),
\end{aligned}
$$

where $\tau_{z}$ is the valley index, $\vec{\sigma}$ and $\vec{s}$ are respectively the sublattice and real spins. The most interesting component is $H_{S O}$, proportional to $\sigma_{z}$ therefore breaking the sublattice symmetry, which can turn graphene into a topological insulator. Calculations based on density functional theory [41, 42], have shown that graphene can inherit strong spin-orbit coupling from a dilute concentration of heavy adatoms randomly deposited onto the honeycomb lattice. The atomic SOI of these elements can be of the order of an electron-Volt. The SOI induced in graphene can be understood by considering processes in which an electron from graphene tunnels onto an adatom where upon it feel the spin-orbit coupling and then returns to the graphene sheet. Depending on the position of the adsorbed atoms with respect to the hexagonal cell, their concentration, and the microscopic nature of the induced SOI, different behaviors have been predicted, from the generation of a spin Hall effect, to a spin orbit band splitting or a spin Hall insulator with spin filtered edge states. Indium and thallium atoms are expected to occupy sites at the center of the hexagons of the honeycomb lattice, transforming graphene into a spin Hall insulator. On the other hand, certain adatoms like osmium and iridium form spin-orbit-split impurity bands that hybridize with graphene Dirac states. It is then more appropriate to view the adatoms as the dominant low-energy degrees of freedom, with their coupling effectively mediated by graphene. However whereas the configuration of diluted atoms has been investigated in detail, the more realistic situation where atoms form clusters or islands on graphene still needs to be investigated theoretically.

It was recently suggested [43] that measuring the ratio $\tau_{t r} / \tau_{e}$ as discussed in section 1 can provide information on the amplitude of the spin orbit coupling. Asmar and Uloa have shown that SOI leads to an important transformation of short range scattering low energy processes in graphene from highly anisotropic (zero back scattering) to more or fully isotropic, depending on the strength of these interactions. This is shown in Figure 7 showing the evolution of the angular dependence of the scattering cross section and $\tau_{t r} / \tau_{e}$ with the strength of $\Delta_{S O}$.

On the experimental side it has turned out that depositing adatoms on graphene in a controlled soft way is not an easy task and can strongly degrade the quality of graphene by the formation of extra defects and clustering.

An alternative approach for inducing spin-orbit coupling in graphene is to functionalize samples with organo-metallic molecules containing heavy non magnetic atoms such as metallo-porphyrins or phtalocyanins. In contrast to evaporated atoms 

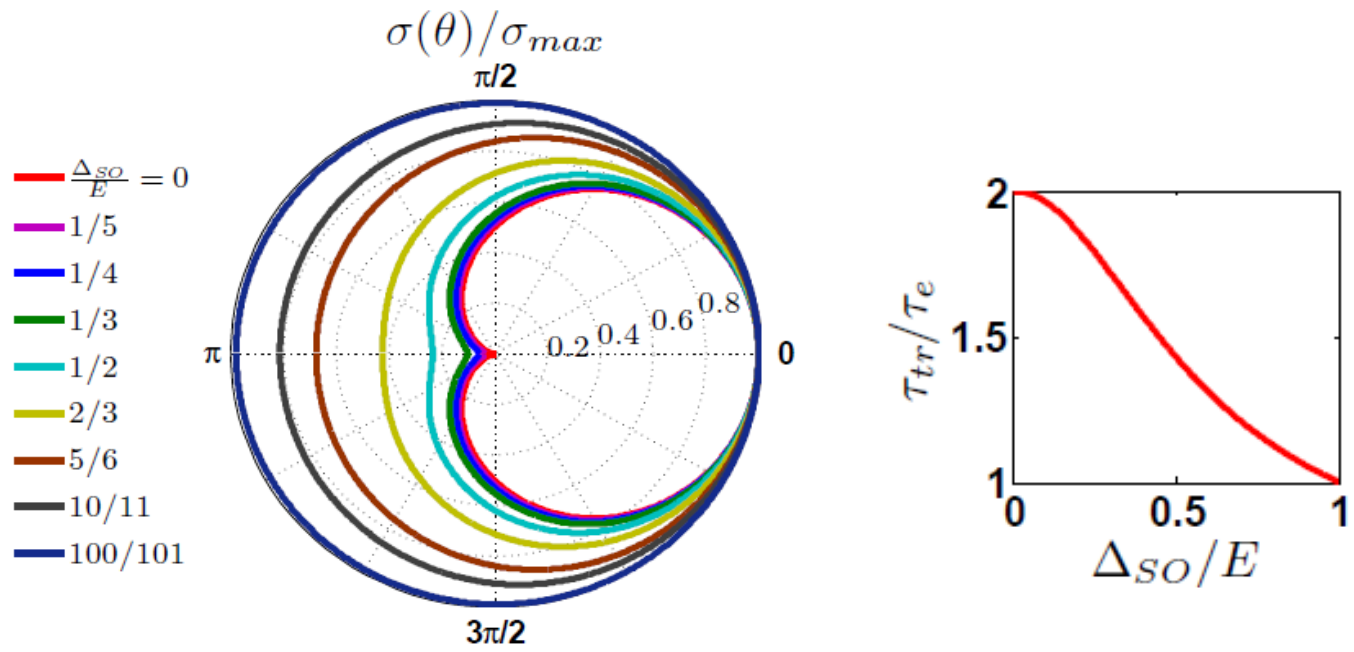

Figure 7: Evolution of the angular dependence of the scattering crossection on resonant impurities with increasing spin orbit strength. On can see that an angular dependence is recovered at large spin orbit coupling which indicates that intravalley back scattering is not forbidden anymore. Inset: evolution of the ratio $\tau_{t r} / \tau_{e}$, which varies between 2 to 1 (from [43]).

which tend to cluster in a disordered way, these molecules are known to form ordered arrays with a periodicity that can be adjusted by choosing the size of the organic side chains of the molecules. Among them, porphyrins and phtalocyanins are already known to self-organize into well-ordered structures on clean graphite surfaces [44]. When deposited on hole doped graphene, these molecules act as donors and electron-dope the pristine substrate. We have found that the mobility can even be increased [40]. On the other hand hole doped doping is observed when graphene is initially electron doped. In general we have found that the molecules tend to neutralise charges on graphene. We have chosen to work with metallo-porphyrin molecules with heavy Pt atoms in order to induce large spin spin-orbit interactions. Whereas the amplitude of induced SOI could not be estimated yet, we found, by using superconducting electrodes, that gate-controlled magnetism can be induced in graphene: Pt porphyrins are non-magnetic when they are neutral, but acquire a magnetic moment when they are ionized. We found that the gate voltage controls this ionization process at room temperature, but not at low temperature. It is thus in principle possible to freeze a given ionization state of the porphyrin molecules on graphene by cooling the sample at a chosen gate voltage. The magnetic state of the ionized porphyrins can be subsequently controlled at low temperature as revealed by proximity-induced superconductivity experiments (see Figure 3). These results may be evidence for the long-sought Fermi-level-controlled exchange interaction between localized spins and graphene, leading to new kinds of gate controlled magnetic devices. Measurements under different field orientations exhibit hysteretic behavior, revealing a strong magnetic anisotropy, a promising observation that needs to be understood. For the purpose of demonstrating a spin orbit interaction induced by these molecules, a probable route will be to adjust the doping at room temperature (via the gate voltage) so that the molecules are not ionized and therefore not magnetic. We expect that in this case the induced spin orbit interaction will overcome 
the magnetic interactions at low temperature.

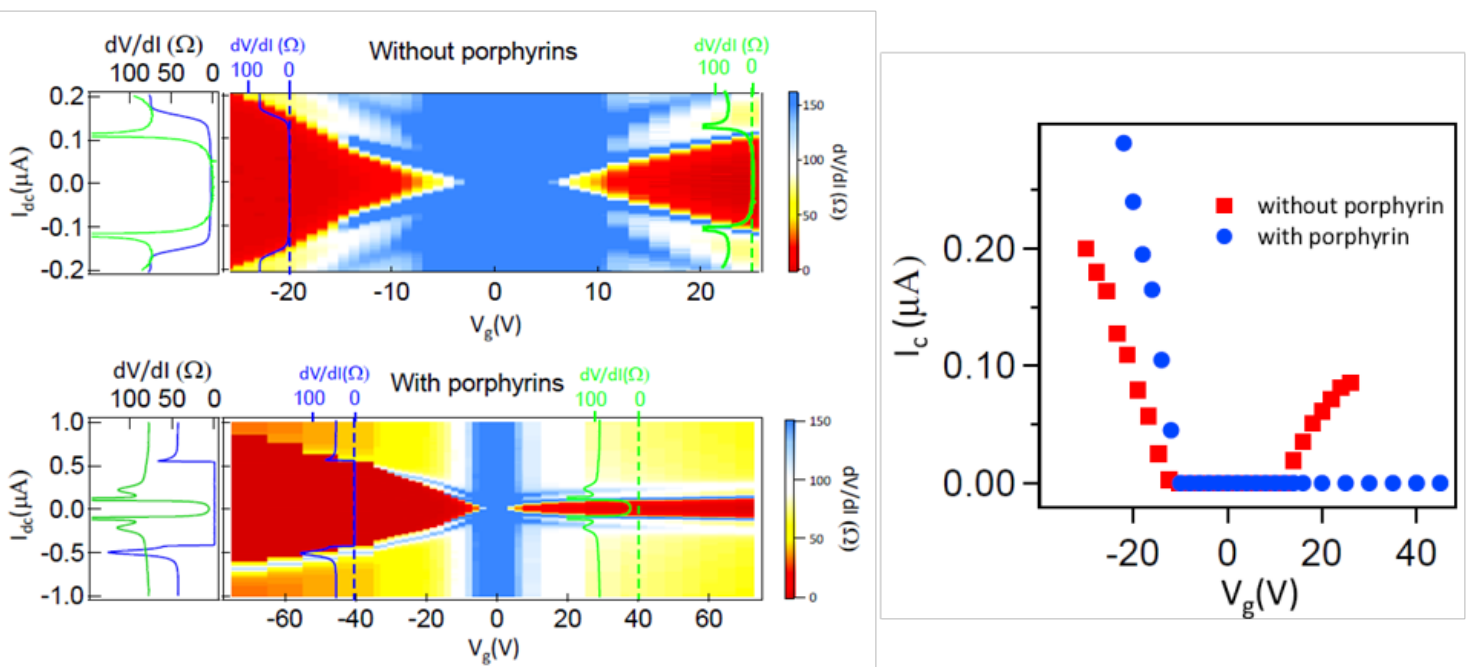

Figure 8: Comparison between the proximity effect in graphene connected to $\mathrm{Pd} / \mathrm{Nb}$ electrodes before (upper) and after (lower) deposition of porphyrin molecules. The color scale codes the differential resistance as a function of gate voltage ( $\mathrm{x}$ axis), measured with a small ac current added to the dc current (y axis). The dark red region corresponds to the region of zero differential resistance where a Josephson supercurrent runs through the S/graphene/S junction. Whereas the Josephson effect occurs symmetrically on both sides of the Dirac point on the bare sample, (bipolar supercurrent) it only occurs on the hole doped side on the sample covered with porphyrins (unipolar supercurrent). The curves on the left of the color plots correspond to the differential resistance curves measured at gate voltages symmetric with respect to the Dirac point. Right: gate voltage dependence of the supercurrent on the bare graphene sample compared to the graphene grafted with porphyins. These findings are interpreted as the signature of the existence of a collective magnetic state for the ionized porphyrins mediated by graphene conduction electrons for positive gate voltages [40].

\subsection{Engineering inhomogeneous strain in graphene}

In contrast with the modification of the electronic properties of graphene by the deposition of adatoms or molecules we discuss now another way to engineer new electronic properties in graphene based on the generation of local strained regions. It was pointed out that new non-trivial electronic properties were to be expected in strained graphene nanostructures in which the two atoms in the unit cell experience different potentials. This effect can be described by a polarization of the pseudo spin degrees of freedom under large effective magnetic fields [11]. We have recently found that it is possible to deposit graphene on etched substrates consisting of arrays of silicon oxide nanopillars (see Fig. 9). We obtain this way a periodic array of graphene strained bumps separated by ripples. Depending on the lattice spacing of the array of pillars, different conformations of graphene were observed ranging from complete suspension of graphene or on the opposite a complete coverage of the substrate with the formation of ripples connecting nearest neighbour pillars. These structures have been investigated in detail in [45] combining electron and atomic force microscopy observations with Raman measurements. Transport properties are 
under investigation. Both strain inhomogeneities and ripples are expected to strongly affect electronic properties of graphene. Whereas a local strain is expected to induce a polarization of the pseudo spin degrees of freedom acting as an effective magnetic field which modifies the density of states, a ripple scatters charge carriers creating a dephasing field within intravalley transport. In the presence of a gate voltage, ripples can behave as smooth pn junctions leading to anisotropic transport and collimation effects [46]. Moreover graphene bubbles can be considered as quantum dots, the confined energy spectrum of which, has been calculated [47]. Depending on the geometry of the bubble, level crossings are found. These give rise to a strongly modified orbital magnetism under an applied (real) magnetic field expected to be paramagnetic rather than diamagnetic. More spectacular is the possible existence of current loops constituting a magnetic texture around the bubble in the absence of any applied magnetic field [48].
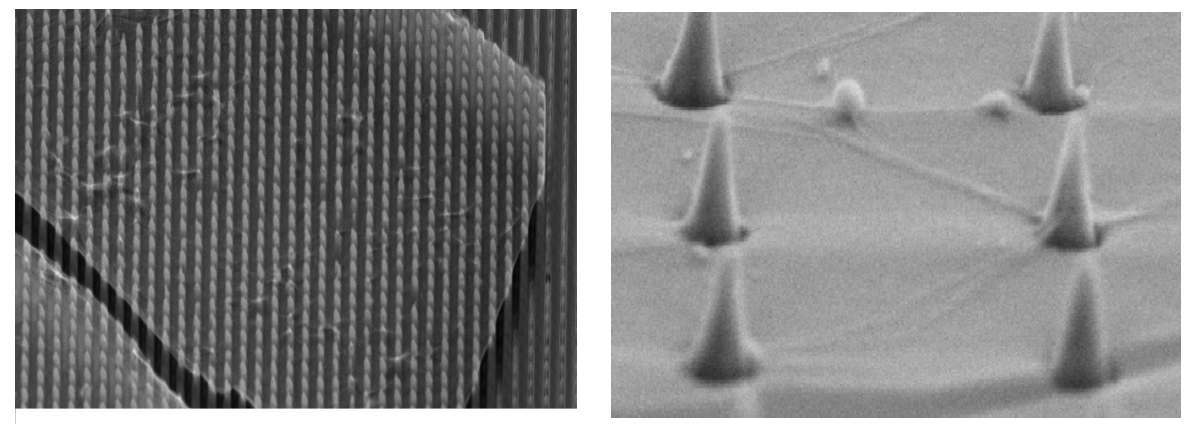

Figure 9: Scanning electron microscopy picture of CVD Graphene stretched on a network of 300nm high $\mathrm{SiO}_{2}$ nanopillars [45]. Examples of graphene transferred on pillar arrays. Work done in collaboration between LPS Orsay and Neel groups leading to partially (left) or totally (right) suspended graphene.

\section{Conclusion}

We have shown that transport properties of graphene in the presence of disorder or intentionally deposited adatoms and molecules, bear the signature of its Dirac particular spectrum as well as the symmetry properties of the wave functions. We have first considered the case of strong resonant scatterers whose range is intermediate between the carbon-carbon nearest neighbour distance $(0.3 \mathrm{~nm})$ in graphene and the Fermi wave length (few $\mathrm{nm}$ ) which play the dominant role in the limitation of the mobility of graphene. Inspite of being short range, these scatterers do not lead to intervalley scattering and are sensitive to the Berry phase factor of the pseudo-spin component of the wave function. In the absence of spin orbit scattering, they are characterized by a ratio $r=\tau_{t r} / \tau_{e}$ equal to 2 measured for graphene instead of the value of 1 obtained for a single parabolic spectrum. It was pointed out theoretically that the value $r=1$ can be recovered for impurities with a large spin orbit scattering potential, suggesting that the investigation of this ratio $\mathrm{r}$ can yield the strength of the the spin Orbit component of the scattering potential. The case of the bilayer still needs to be understood. In the opposite limit of very long 
range scatterers, the second section of this paper was devoted to the sensitivity of quantum transport to the formation of electron hole puddles in the low doping region. A spectacular experimental consequence is the complete suppression of the supercurrent close to the Dirac point in long graphene based Josephson junctions. We also have shown that functionalised graphene with grafted porphyrin molecules lead to new properties such as gate voltage dependent magnetism and potentially spin spin orbit interactions. The possibility to induce periodic inhomogeneous strain networks is also discussed.

\section{Acknowledgments}

We thank all our colleagues who largely contributed to the work presented in this paper: Miguel Monteverde, Claudia Ojeda, Jean Noel Fuchs, Katsuyoshi Komatsu, Dimitri Maslov, Keyan Bennaceur, Christian Glattli, Raphael Weil, Sandrine AutierLaurent.

\section{References}

[1] Castro Neto, A. H., et al.: Rev. Mod. Phys. 81, 109 (2009).

[2] Wallace, P. R.: Phys. Rev. 71, 622 (1947).

[3] Shon, N., and Ando, T.: J. Phys. Soc. Jpn 67, 2421 (1998).

[4] Akkermans, E., and Montambaux, G.: Mesoscopic Physics with electrons and photons. Cambridge University Press, (2007).

[5] Coleridge, P. T.: Phys. Rev. B 44, 3793 (1991).

[6] Ojeda-Aristizabal, C., Monteverde, M., Weil, R., Ferrier, M., Guéron, S., and Bouchiat, H.: Phys. Rev. Lett. 104, 186802 (2010).

[7] Heersche, H. B., et al.: Nature 56, 446 (2007).

[8] Du, X., Skachko, I., and Andrei, E. Y.: Phys. Rev. B 77, 184507 (2008).

[9] Ojeda-Aristizabal, C., Ferrier, M., Guéron, S., and Bouchiat, H.: Phys. Rev. B 79, 165436 (2009).

[10] Dongchan Jeong, Jae-Hyun Choi, Gil-Ho Lee, Sanghyun Jo, Yong-Joo Doh, and Hu-Jong Lee: Phys. Rev. B 83, 094503 (2011).

[11] Levy, N., Burke, S. A., Meaker, K. L., Panlasigui, M., Zettl, A., Guinea, F., Castro Neto, A. H., and Crommie, M. F.: Science 329, 544 (2010).

[12] Aleiner, I. L., and Efetov, K. B.: Phys. Rev. Lett. 97, 236801 (2006).

[13] Ostrovsky, P. M., Gornyi, I. V., and Mirlin, A. D.: Phys. Rev. B 74, 235443 (2006).

[14] Nomura, K., MacDonald, A. H.: Phys. Rev. Lett. 96, 256602 (2006); Ando, T.: J. Phys. Soc. Japan 75, 074716 (2006).

[15] Adam, S., and das Sarma, S.: Phys. Rev. B 77,115436 (2008). 
[16] Adam, S., Cho, S., Fuhrer, M., and Das Sarma, S.: Phys. Rev. Lett. 101, 046404 (2008).

[17] Jang, C., et al.: Phys. Rev. Lett. 101, 146805 (2008); Ponomarenko, L. A., et al.: Phys. Rev. Lett. 102, 206603 (2009).

[18] Couto, N. J. G., Sacepe, B., and Morpurgo, A. F.: Phys. Rev. Lett. 107, 225501 (2011).

[19] Katsnelson, M. I., and Novoselov, K. S.: Solid State Commun. 143, 3 (2007); Stauber, T., Peres, N. M. T., and Guinea, F.: Phys. Rev. B 76, 1120 (2007).

[20] Cho, S., and Fuhrer, M. S.: Phys. Rev. B 77, 081402 (2008).

[21] Abanin, D. A., and Levitov, L. S.: Phys. Rev. B 78, 035416 (2008).

[22] Williams, J. R., et al.: Phys. Rev. B 80, 045408 (2009).

[23] Lifshitz, I.M., and Kosevich, A.M.: Sov. Phys. JETP 2, 636 (1956); Coleridge, P.T., Stoner, R., and Fletcher, R.: Phys. Rev. B 39, 195412 (1989).

[24] Monteverde, M., Ojeda-Aristizabal, C., Weil, R., Bennaceur, K., Ferrier, M., Guéron, S., Glattli, C., Bouchiat, H., Fuchs, J. N., and Maslov, D. L.: Phys. Rev. Lett. 104, 126801 (2010).

[25] Hwang, E. H., and Das Sarma, S.: Phys. Rev. B 77, 195412 (2008).

[26] Adhikari, S. K.: Am. J. Phys. 54, 362 (1986).

[27] Novikov, D. S.: Phys. Rev. B 76, 245435 (2007).

[28] Basko, D.: Phys. Rev. B 78, 115432 (2008).

[29] Meyer, J. C., et al.: Nano Lett. 8, 3582 (2008).

[30] Katsnelson, M. I., Vozmediano, M. A., and Guinea, F.: Phys. Rev. B 77, 075422 (2008).

[31] Couto, N. J. G., et al.: arXiv:1401.5356.

[32] Tikhonenko, F. V., Horsell, D. W., Gorbachev, R. V., Savchenko, A. K.: Phys. Rev. Lett. 100, 056802 (2008).

[33] Martin, J., et al.: Nat. Phys. 4, 144 (2008).

[34] Beenakker, C. W. J.: Rev. Mod. Phys. 80, 1337 (2008).

[35] Titov, M., and Beenakker, C. W. J.: Phys. Rev. B 74, 041401(R) (2006).

[36] Katsuyoshi, Komatsu Chuan, Li, Autier-Laurent, S., Bouchiat, H., and Guéron, S.: Phys. Rev. B 88, 115412 (2012).

[37] Yang Wang, et al.: Science 340, 734 (2013).

[38] Uchoa, B., Kotov, Valeri N., Peres, N. M. R., and Castro Neto, A. H.: Phys. Rev. Lett. 101, 026805 (2008).

[39] Kotov, Valeri N., Uchoa, B., Pereira, Vitor M., Castro Neto, A.H., et al.: Rev. Mod. Phys. 84,1067 (2012). 
[40] Chuan Li, et al.: arXiv:1304.7089.

[41] Weeks, C., Hu, J., Alicea, J., Franz, M., and Wu, R.: Phys. Rev. X 1, 021001 (2011).

[42] Hu, J., Alicea, J., Wu, R., and Franz, M.: Phys. Rev. Lett. 109, 266801 (2012).

[43] Asmar, Mahmoud M., Ulloa, Sergio E.: Phys. Rev. Lett. (2014).

[44] Otsuki, J., Kawaguchi, S., Yamakawa, T., Asakawa, M., and Miyake, K.: Langmuir 22, 5708-5715 (2006).

[45] Reserbat-Plantey, A., et al.: arXiv:1404.5783.

[46] Cheol-Hwan Park, Young-Woo Son, Li Yang, Cohen, Marvin L., Louie, Steven G.: Nano Lett. 8, 2920-2924 (2008).

[47] Kyung-Joong Kim, Blanter, Ya. M., and Kang-Hun Ahn: Phys. Rev. B 84, 081401 (2011).

[48] Guinea, F., Katsnelson, M. I., and Geim, A. K.: Nature Physics 6, 30-33 (2010);

Wakker, G. M. M., et al.: Phys. Rev. B 84, 195427 (2011). 